\title{
A CNN-based hybrid model to detect Coronavirus disease
}

\author{
Ebru Erdem ${ }^{1 *}$, Tolga Aydin ${ }^{2}$ \\ 1* Ataturk University, Faculty of Engineering, Department of Computer Engineering, Erzurum, Turkey, (ORCID: 0000-0002-4042-7549), ebruerdem@atauni.edu.tr \\ ${ }^{2}$ Ataturk University, Faculty of Engineering, Department of Computer Engineering, Erzurum, Turkey, (ORCID: 0000-0002-8971-3255), atolga@atauni.edu.tr
}

(First received 12 May 2021 and in final form 15 August 2021)

(DOI: $10.31590 /$ ejosat.936820)

ATIF/REFERENCE: Erdem, E. \& Aydın, T. (2021). A CNN-based hybrid model to detect Coronavirus disease. European Journal of Science and Technology, (27), 66-73.

\begin{abstract}
In this paper, a hybrid classification technique for COVID-19 disease is proposed. The proposed model solves the two-class classification problem (covid, normal). In this study, we have presented hybrid models integrating superior deep learning and machine learning classifiers: Convolutional Neural Network (CNN) and Support Vector Machine (SVM), CNN and AdaBoost, CNN and K Nearest Neighbour (kNN), CNN and Multilayer Perceptron (MLP), CNN and Naive Bayes (NB). In these models, CNN performs as a trainable deep feature extractor, and SVM, AdaBoost, kNN, MLP, NB behave as recognizers. All experiments have been performed on COVID-CT and SARS-CoV-2 CT combined image datasets. As a result, proposed hybrid methods have been compared in terms of sensitivity, accuracy, precision, F1-score, AUC-score, specificity, FPR, FDR, and FNR. CNN+SVM, CNN+MLP, and CNN+kNN have achieved outperforming results according to the other models. Also, CNN+SVM performed the best. When the results are examined, the proposed hybrid system is seen to be efficient to detect COVID-19. Also, the performance of the proposed hybrid system is better than the successful studies found on COVID-CT and SARS-CoV-2 CT combined image datasets in the literature.
\end{abstract}

Keywords: Covid-19, hybrid models, deep learning, CNN.

\section{Koronavirüs hastalığını tespit etmek için CNN tabanlı bir hibrit model}

$\ddot{O} \mathbf{z}$

Bu yazıda, COVID-19 hastalığı için hibrit bir sınıflandırma tekniği önerilmektedir. Önerilen model, iki sınıflı sınıflandırma problemini çözmektedir (covid, normal). Bu çalışmada, üstün derin öğrenme ve makine öğrenimi sınıflandırıcılarını entegre eden hibrit modeller sunduk: Evrişimsel Sinir Ağ (CNN) ve Karar Destek Makinesi (SVM), CNN ve AdaBoost, CNN ve K En Yakın Komşu (kNN), CNN ve Çok Katmanlı Algılayıcı (MLP), CNN ve Naive Bayes (NB). Bu modellerde CNN, eğitilebilir bir derin özellik çıkarıcı olarak çalışır ve SVM, AdaBoost, kNN, MLP, NB bir tanıyııı olarak davranır. Tüm deneyler, COVID-CT ve SARSCoV-2 CT birleşik görüntü veri kümeleri üzerinde gerçekleştirilmiştir. Sonuç olarak, önerilen hibrit yöntemler duyarlıllk, doğruluk, kesinlik, F1 puanı, AUC puanı, özgüllük, FPR, FDR ve FNR açısından karşılaştırılmıştır. CNN + SVM, CNN + MLP ve CNN + kNN, diğer modellere göre sırasıyla daha iyi performans gösteren sonuçlar elde etmiştir. Ayrıca, CNN + SVM en iyi performansı göstermiştir. Sonuçlar incelendiğinde, önerilen hibrit sistemin COVID-19'u tespit etmede etkili olduğu görülmektedir. Ayrıca, önerilen hibrit sistemin performansı, literatürdeki COVID-CT ve SARS-CoV-2 CT birleşik görüntü veri kümelerinde bulunan başarılı çalışmalardan daha iyidir.

Anahtar Kelimeler: Covid-19, hibrit modeller, derin öğrenme, CNN.

\footnotetext{
* Corresponding Author: ebruerdem@ atauni.edu.tr
} 


\section{Introduction}

At the last days of 2019, Coronavirus disease of 2019 (COVID-19) has emerged in China and quickly disseminated to the globe. The number of infected cases is increasing rapidly in many countries. Early diagnosis of disease is very important in terms of quarantine of infected cases (KARAKUŞ, 2020).

The clinical symptoms (cough, shortness of breath, etc.) of the disease are not specific, so the detection progress is challenging. The reverse-transcription polymerase chain reaction (RT-PCR) test is a generally employed standard technique to detect the disease. However, it has many shortcomings including resulting in false-negatives and being time-consuming.

Computed tomography (CT) is a well-known imaging technique for a successful and early recognition of the disease. Thus, radiological imaging is important as a diagnostic tool. The computer-aided design (CAD) systems that use CT images are recommended for the control and the interpretation of the disease case. These systems are critical for helping radiologists in accurately diagnosing the disease based on medical image data.

Numerous articles have been published in the literature recently. Especially, the classification of the CT images was conducted via artificial intelligence techniques and successful results have been obtained, as the following paragraph discusses.

In their study, Wu et al. applied ResNet50 on 368 COVID19 CT images from Beijing Youan Hospital and China Medical University. They achieved accuracy, sensitivity, specificity, and AUC rates of $76 \%, 81.1 \%, 61.5 \%$, and $81.9 \%$, respectively (Wu et al., 2019). Jin et al. applied ResNet152 on 1881 COVID-19 CT images from Wuhan Union Hospital, Jianghan Mobile Cabin Hospital, and Western Campus of Wuhan Union Hospital. They achieved accuracy, sensitivity, specificity, precision, F1-score, and AUC rates of $94.98 \%, 94.06 \%, 95.47 \%, 91.53 \%, 92.78 \%$, and $97.91 \%$, respectively (Jin et al., 2020). Javaheri et al. applied BCDU-Net on 32230 covid data from five medical centers. They achieved accuracy, sensitivity, specificity, and AUC rates of $91.66 \%, 87.5 \%, 94 \%$, and $95 \%$, respectively (Javaheri et al., 2020). Jin et al. applied different DL based algorithms (DPN-92, Inception-v3, ResNet-50, Attention ResNet50 with 3D U-Net++) on 1391 COVID-19 CT images from five different hospitals of China. They achieved AUC, sensitivity, and specificity rates of $99.1 \%, 97.04 \%$, and $92.2 \%$, respectively (Jin et al., 2020). Chen et al. applied Unet++ on 35355 CT images from the Renmin Hospital of Wuhan University. They achieved accuracy, sensitivity, specificity, precision, and AUC rates of 98.85\%, 94.34\%, 99.16\%, 88.37\%, and $99.4 \%$, respectively (Chen et al., 2020). Ardakani et al. applied DL based algorithms (AlexNet, VGG-16, VGG-19, SqueezeNet, GoogleNet, MobileNet-V2, ResNet-18, ResNet-50, ResNet-101, Xception) on 510 CT images from real-time data. They achieved accuracy, sensitivity, specificity, precision, and AUC rates of $99.51 \%, 100 \%, 99.02 \%, 99.27 \%$, and $99.4 \%$, respectively (Ardakani et al., 2020). He and his colleagues proposed CRNet in their work. With this model, they have reached an $86 \%$ accuracy rate, $85 \% \mathrm{~F} 1$-score, and 94\% AUC (He et al., 2020). Wang et al. proposed the Modified-Inception model for classification. They have reached $79.3 \%$ accuracy rate, $83 \%$ sensitivity, $67 \%$ specificity, $55 \%$ precision, $63 \% \mathrm{~F} 1-$ score, and $81 \%$ AUC (Wang et al., 2020). Ying et al. suggested DRE-Net on 777 COVID positive CT data from the Third Affiliated Hospital and Hospital of Wuhan University. With this model, they have reached $94.3 \%$ accuracy rate, $93 \%$ sensitivity rate, $96 \%$ precision rate, 94\% F1-score, and 99\% AUC (Song et al., 2020). Zheng et al. applied DeCoVNet on 630 CT images from three different hospitals (Huazhong University of Science and Technology, Union Hospital, and Tongji Medical College). They achieved accuracy, sensitivity, specificity, precision, and AUC rates of $90.1 \%, 90.7 \%, 91.1 \%, 84 \%$, and $95.9 \%$, respectively (Zheng et al., 2020). Singh et al. proposed MODECNN in their work. With this model, they have reached a $93.25 \%$ accuracy rate, $90.70 \%$ sensitivity rate, $90.72 \%$ specificity rate, and $89.96 \%$ F1-score (Singh et al., 2020). Farid et al. applied a CNN model on 51 COVID-19 CT images from the Kaggle benchmark dataset. They achieved accuracy, precision, F1-score and AUC rates of $94.11 \%, 99.4 \%, 94 \%$, and $99.4 \%$, respectively (Farid et al., 2020). The writers in Wang et al. suggested employing the Inception and Adaboosted decision tree framework. They recognized COVID-19 cases achieving an accuracy of $82.9 \%$, an AUC of $90 \%$, a sensitivity of $81 \%$, and a specificity of $84 \%$ (Wang et al., 2020). The writers in Song et al. presented a ResNet-50 architecture. The accuracy, AUC, sensitivity, and precision values proved to be $86 \%, 95 \%, 96 \%$, and $79 \%$, respectively (Song et al., 2020). The researchers in Jin et al. used the Deeplab-v1 and ResNet-152 deep learning framework to make a distinction between COVID-19 and nonCOVID-19 cases. An accuracy of $94.8 \%$, an AUC of $97.9 \%$, a sensitivity of $94.1 \%$, and a specificity of $95.5 \%$ were obtained (Jin et al., 2020).

Other studies proposed DL-based architectures for novel coronavirus pneumonia (NCP) diagnosis. However, as it is observed, the effectiveness of CNN-based deep hybrid approaches can more be required in the literature. Therefore, in this paper, $\mathrm{CNN}$ architecture was used as a basic network for a hybrid model approach.

\section{Material and Method}

\subsection{Material}

Two public datasets are used to predict and evaluate our framework, including COVID-CT (Yang et al., 2020; 2020) and SARS-CoV-2 CT-scan image datasets (Soares et al., 2020; 2020) (Fig. 1). 

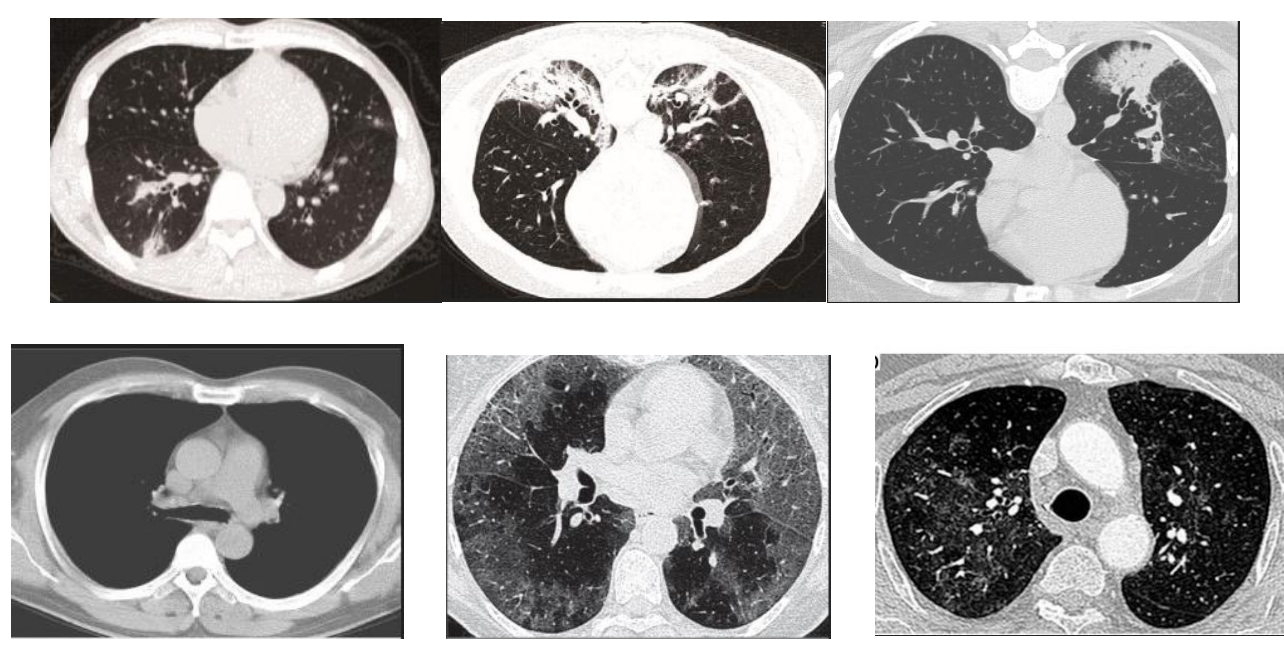

Figure 1 Examples of CT Images (COVID-19 (Top) and Non-COVID-19 (Bottom))

SARS-CoV-2 CT-scan dataset is composed of CT scan images that were collected from hospitals in Brazil. This dataset consists of $2482 \mathrm{CT}$ scans from 120 cases, with $1252 \mathrm{CT}$ scans of 60 cases infected and $1230 \mathrm{CT}$ scans of 60 cases non-infected by SARS-CoV-2. COVID-CT dataset is composed of CT images of patients infected with SARS-CoV-2, These were obtained from the research articles. This dataset consists of 349 CT scans from 216 patients. Metadata is associated with properties like patient gender, age, scan time, the severity of the disease, etc. Regarding to non-covid patients, this dataset consists of $463 \mathrm{CT}$ scans from 55 patients. These images were collected from websites, articles, and other resources.

In this paper, SARS-CoV-2 CT-scan and COVID-CT images are combined. The combined dataset consists of $3227 \mathrm{CT}$ images, with 1601 covid and 1626 non-covid cases.

\subsection{The proposed framework}

In this study, five different DL-based hybrid models are applied to predict and evaluate the classification results. These models are used on the combined image dataset.

The proposed hybrid system uses the deep features derived from CNN separately and utilizes these features to train SVM, AdaBoost, kNN, MLP, and NB classifiers. Then, the classification performance of the classifiers is evaluated (Fig. 2).

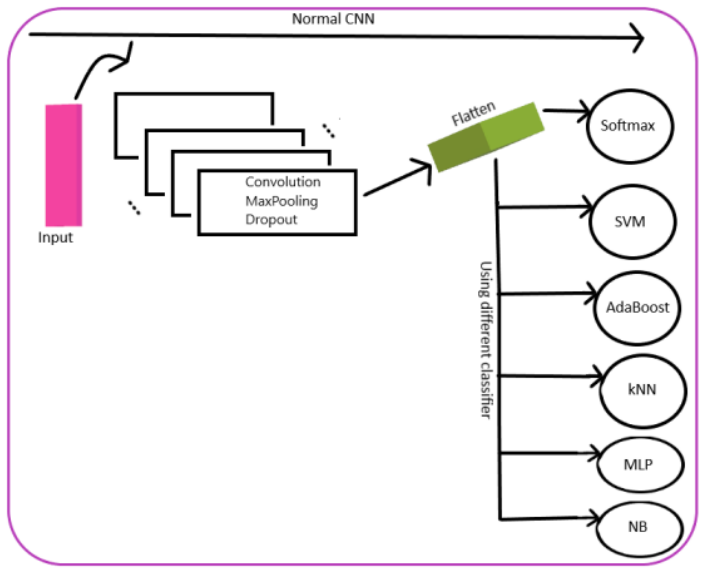

Figure 2 The Architecture of The DL Based-CAD System

A convolutional neural network is one of the deep learning architectures. This architecture is usually composed of the e-ISSN: 2148-2683 following types of layers: convolutional, activation, dropout, pooling, and softmax (Fig. 3). Convolutional neural networks vary in how convolutional and other sublayers are trained and implemented.

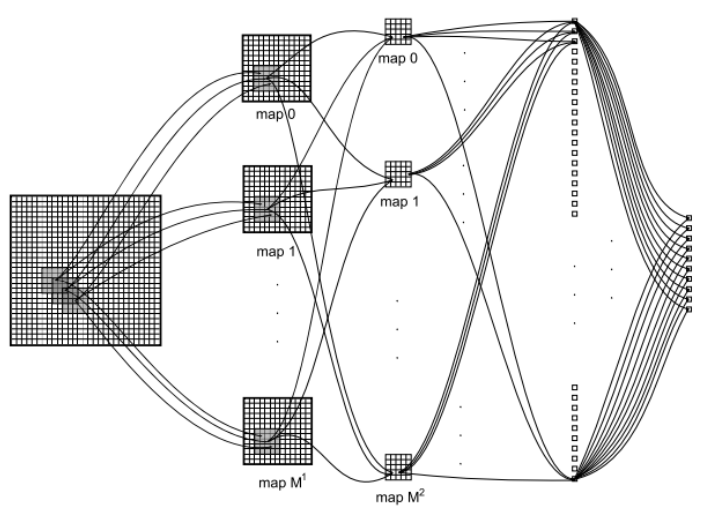

Figure 3 General Architecture of A CNN [Ciresan et al., 2011]

The convolutional layer provides producing feature maps. This layer has trainable bias and trainable filters per feature map. Each output map is connected to all of its preceding feature maps. The convolution process is presented in Eq. (1) (Yildirim \& Cinar, 2020). In Eq. (1); x, w, t, and s represents the input, filter, times, and result, respectively.

$$
s(t)=(x * w)(t)=\sum_{n=-\infty}^{\infty}(x(n)+w(t-n))
$$

Activation functions are important to help the model to learn better. The activation function is usually used for nonlinear transformation. Relu was preferred for this study (Eq. (2)) (Yildirim \& Cinar, 2020). The obtained output feature maps with the convolutional layer are passed to this layer.

$$
f(x)=\left\{\begin{array}{l}
0, x<0 \\
x, x \geq 0
\end{array}, f(x)^{\prime}= \begin{cases}0, & x<0 \\
1, & x \geq 0\end{cases}\right.
$$

Then, while moving to the next layer, a maximum pooling process is applied to reduce the spatial size of the input (Eq. (3) (Yildirim \& Cinar, 2020)). In Eq. (3); w, h, and d represents width, height, and depth of input image size, respectively. Also, the dropout method was used to improve the performance of the 
model. This technique prevents overfitting. The softmax layer is used to predict the classification label for the final result.

$$
S=w * h * d
$$

$\mathrm{CNN}$ architecture has been used as the basic structure in the hybrid models. The improved hybrid model structure was presented in Fig. 2. At the last layer of the CNN, instead of the softmax function, SVM, AdaBoost, kNN, MLP, and NB were implemented, respectively.

For consistency, all the images are resized to $256 \times 256 \times 3$. That is, the image input sizes are updated as $256 * 256$ with three channels. CNN model was used for the feature extraction. Details of CNN architecture are presented in Table 1.

Features were obtained from the Flatten layer of the CNN architecture. The box plot illustration of the features obtained from the CNN model is given in Fig. 4. The feature values from the $\mathrm{CNN}$ model were in a wide range. Also, the box plot illustration of the value of each feature is given in Fig. 5.

Table 1 Details of Layers Used in CNN

\begin{tabular}{|l|l|l|l|l|l|l|l|}
\hline Layer & Stride & $\begin{array}{l}\text { Filter } \\
\text { size }\end{array}$ & Pool size & Padding & Activation & $\begin{array}{l}\text { Data } \\
\text { depth }\end{array}$ & $\begin{array}{l}\text { No. of } \\
\text { parameters }\end{array}$ \\
\hline Input Layer & - & - & - & - & - & 3 & 0 \\
\hline Conv1 & 1 & $5 \times 5$ & - & Same & Relu & 16 & 1216 \\
\hline MaxPooling & 2 & - & $2 \times 2$ & - & - & 16 & 0 \\
\hline Dropout & - & - & - & - & - & 16 & 0 \\
\hline Conv1 & 1 & $5 \times 5$ & - & Same & Relu & 32 & 12832 \\
\hline MaxPooling & 2 & - & $2 \times 2$ & - & - & 32 & 0 \\
\hline Dropout & - & - & - & - & - & 32 & 0 \\
\hline Conv1 & 1 & $5 \times 5$ & - & Same & Relu & 64 & 51264 \\
\hline MaxPooling & 2 & - & $2 \times 2$ & - & - & 64 & 0 \\
\hline Dropout & - & - & - & - & - & 64 & 0 \\
\hline Conv1 & 1 & $5 \times 5$ & - & Same & Relu & 64 & 102464 \\
\hline MaxPooling & 2 & - & $2 \times 2$ & - & - & 64 & 0 \\
\hline Dropout & - & - & - & - & - & 64 & 0 \\
\hline Flatten & - & - & - & - & - & 64 & 0 \\
\hline
\end{tabular}

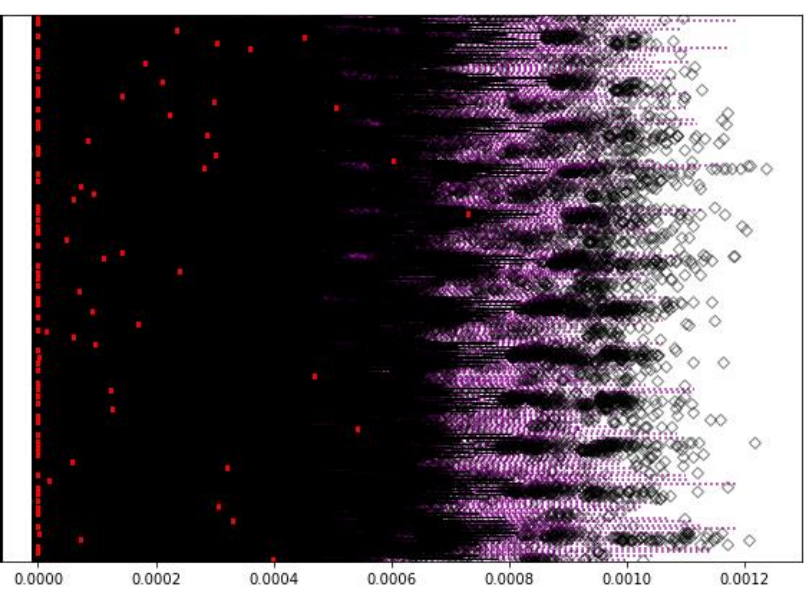

Figure 4 The Illustration of the Feature Values from CNN Model

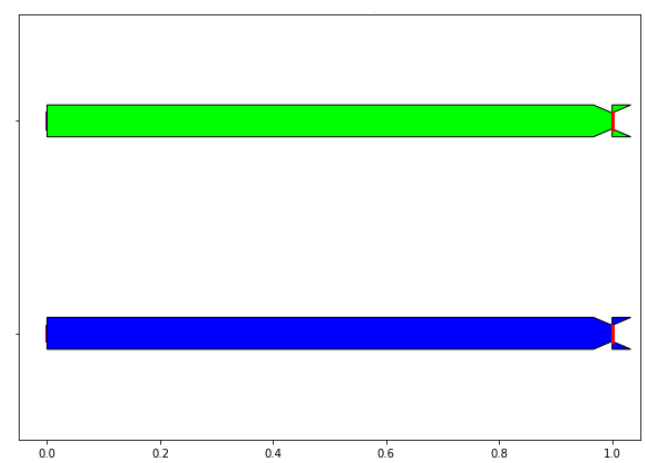

Figure 5 The Illustration of the Target Feature from CNN Model

Later, these features are used in the classification stage of the proposed framework. The classification stage uses 5 different machine learning classifiers. These are SVM, AdaBoost, kNN, MLP, and NB algorithms (Fig. 2). 


\subsection{Support vector machine, Adaptative boosting, K nearest neighbor, Multilayer perceptron, Naive bayes}

The support vector machine (SVM) was first introduced by Vapnik (Cortes, 1995) for classification and regression. The basic idea of the algorithm is to find the best hyperplane (Eq. (4)). In Eq. (4); w, $x, b$, and $f(x)$ represents a vector to the hyperplane, training data, bias, and class, respectively for a twoclass problem.

$$
f(x)=(w * x)+b\left(x \in \mathrm{R}^{\mathrm{m}}\right)
$$

Also, this method is proposed to solve multi-class problems. If the data cannot be separated linearly, a mapping function, called as kernel, is used to determine the hyperplane. So, the hyperplane can be formulated as in Eq. (5). In Eq. (6); $\mathrm{f}\left(\mathrm{x}_{\mathrm{d}}\right), x_{j}$, and $x_{d}$ represents the class of new input data, a support vector, and input data, respectively.

$$
\begin{gathered}
\min \frac{1}{2}\|w\|^{2}=\min \frac{1}{2} \mathrm{w}^{\mathrm{T}} \mathrm{w} \\
\mathrm{f}\left(\mathrm{x}_{\mathrm{d}}\right)=\sum_{j=1}^{N s} a_{j} y_{j} x_{j} x_{d}+b
\end{gathered}
$$

Adaptative boosting (AdaBoost) was proposed by Freund and Schapire in 1996 (Freund \& Schapire, 1997). It was developed for binary classification. This algorithm is an ensemble method and aims to convert a number of weak classifiers into a strong classifier. The final classifier is given in Eq. (7). In Eq. (7); $f_{i}(x), w_{i}$, and $t$ represents the $\mathrm{i}^{\text {th }}$ weak classifier, the weight, and the number of the weak classifiers, respectively.

$$
f(x)= \begin{cases}1, & \text { if } \sum_{i=1}^{t} w_{i} f_{i}(x) \geq \text { threshold } \\ 0, & \text { otherwise }\end{cases}
$$

In the pattern recognition field, K Nearest Neighbor $(\mathrm{kNN})$ is one of the non-parametric classifiers (Cover \& Hart, 1967). The kNN classifier often uses the Euclidean distance metric for measuring the distances between the data points (Eq. (8)) (Witten \& Frank, 2002).

$$
\mathrm{D}(\mathrm{i}, \mathrm{j})=\sqrt{\sum_{t=1}^{m}\left(x_{i t}-x_{j t}\right)^{2}}
$$

The multilayer perceptron (MLP) (Witten \& Frank, 2002) has three layers: input, hidden, and output. It has neurons that map the input to the output class. The output $y_{t}$ of a neuron $t$ is given in Eq. (9). In Eq. (9); $x_{i}, w_{t i}$, and $\varphi($.$) represents the$ input, the connection weight, and the activation function, respectively. In the input layer, the number of neurons is determined by the dimension of the input. In the output layer, the number of neurons is determined by the number of output classes. In the hidden layer, the number of the neurons is determined according to the best performance.

$$
y_{t}=\varphi\left(\sum_{i=0}^{m} x_{i} w_{t i}\right)
$$

The Naive Bayes (NB) algorithm is based on Bayes Theorem (Russell \& Norvig, 2002). Bayes Theorem is given in Eq. (10). In Eq. (10); $P(Y), P(X \mid Y), P(X)$, and $P(Y \mid X)$ represents the probability of the class, conditional probability of the class for the given attribute, probability of the attribute, and the conditional probability that attribute belongs to the class, respectively.

$$
P(Y \mid X)=\frac{P(Y) P(X \mid Y)}{P(X)}
$$

\section{Results}

In this study, all the research is conducted by using Python language using a computer having an Intel(R) Core(TM) i7-7700 CPU, 24 GB memory, and GeForce GT 730 GPU (NVIDIA).

CT images were obtained for the disease detection. The training and test sets were randomly selected as $70 \%$ training and $30 \%$ test data based on the CT image data to analyze the classifier's accuracy and performance.

\subsection{Evaluation metrics}

The performance of the proposed hybrid frameworks was measured with different metrics such as sensitivity, precision, accuracy, F1-score, AUC-score, specificity, FPR, FDR, and FNR. The formulations are shown below: (Eqs. (11) - (18)).

$$
\begin{gathered}
\text { Accuracy }=\frac{\mathrm{TP}+\mathrm{TN}}{\mathrm{TP}+\mathrm{TN}+\mathrm{FP}+\mathrm{FN}} \\
\text { Sensitivity }=\frac{\mathrm{TP}}{\mathrm{TP}+\mathrm{FN}} \\
\text { Specificity }=\frac{\mathrm{TN}}{\mathrm{TN}+\mathrm{FP}} \\
\text { Precision }=\frac{\mathrm{TP}}{\mathrm{TP}+\mathrm{FP}} \\
F 1-\text { score }=\frac{2 \times \mathrm{precision} \times \mathrm{sensitivity}}{\mathrm{TP}+\mathrm{TN}+\mathrm{FP}+\mathrm{FN}} \\
F P R=\frac{\mathrm{FP}}{\mathrm{FP}+\mathrm{TN}} \\
F D R=\frac{\mathrm{FP}}{\mathrm{FP}+\mathrm{TP}} \\
F N R=\frac{\mathrm{FN}}{\mathrm{FN}+\mathrm{TP}}
\end{gathered}
$$

In these equations, four statistical indices are calculated; namely, false negative (FN), true negative (TN), false positive (FP), and true positive (TP). FN is the number of covid images that are mistakenly classified as non-covid. $\mathrm{TN}$ is the number of non-covid images that are correctly classified. FP is the number of non-covid images mistakenly classified as Covid-19. TP is the number of covid images that are correctly classified. 
Table 2 Performance of DL-based Hybrid Models

\begin{tabular}{|c|c|c|c|c|c|c|c|c|c|c|}
\hline Method & Class & $\begin{array}{c}\text { Sensitivity } \\
(\%)\end{array}$ & $\begin{array}{c}\text { Precision } \\
(\%)\end{array}$ & $\begin{array}{c}\text { Accuracy } \\
(\%)\end{array}$ & $\begin{array}{c}\text { F1-score } \\
(\%)\end{array}$ & $\begin{array}{c}\text { AUC-score } \\
(\%)\end{array}$ & $\begin{array}{c}\text { Specificity } \\
(\%)\end{array}$ & $\begin{array}{c}\text { FPR } \\
(\%)\end{array}$ & $\begin{array}{c}\text { FDR } \\
(\%)\end{array}$ & $\begin{array}{c}\text { FNR } \\
(\%)\end{array}$ \\
\hline $\mathrm{CNN}+\mathrm{SVM}$ & covid & 85.85 & 85.86 & 85.86 & 85.85 & 85.85 & 86.47 & 13.52 & 13.86 & 14.76 \\
\hline CNN+AdaBoost & covid & 73.68 & 73.68 & 73.68 & 73.68 & 73.68 & 73.77 & 26.22 & 26.55 & 26.40 \\
\hline CNN+kNN & covid & 82.25 & 82.26 & 82.24 & 82.24 & 82.25 & 81.14 & 18.85 & 18.66 & 16.63 \\
\hline CNN+MLP & covid & 82.35 & 82.35 & 82.35 & 82.35 & 82.35 & 81.96 & 18.03 & 18.10 & 17.25 \\
\hline
\end{tabular}

The performance results of the proposed framework are shown in Table 2.

$\mathrm{CNN}+\mathrm{SVM}$ performed the highest with a sensitivity of $85.85 \%$, a precision of $85.86 \%$, an accuracy of $85.86 \%$, an F1score of $85.85 \%$, an AUC score of $85.85 \%$, a specificity of $86.47 \%$, an FPR of $13.52 \%$, an FDR of $13.86 \%$, and an FNR of $14.76 \%$.

$\mathrm{CNN}+\mathrm{NB}$ achieved the poorest performance with a sensitivity of $53.29 \%$, a precision of $56.47 \%$, an accuracy of $53.04 \%$, an F1-score of $46.62 \%$, an AUC score of $53.29 \%$, a specificity of $18.23 \%$, an FPR of $81.76 \%$, an FDR of $48.42 \%$, and an FNR of $11.64 \%$.

CNN+MLP model demonstrated higher accuracy $(82.35 \%)$ than $\mathrm{CNN}+\mathrm{kNN}(82.26 \%)$ and $\mathrm{CNN}+\mathrm{AdaBoost}(73.68 \%)$. Also, with respect to sensitivity, precision, F1-score, AUC score, and specificity, the results show that the CNN+MLP model achieved higher performance than $\mathrm{CNN}+\mathrm{kNN}$ and $\mathrm{CNN}+\mathrm{AdaBoost}$ (Fig. 6).

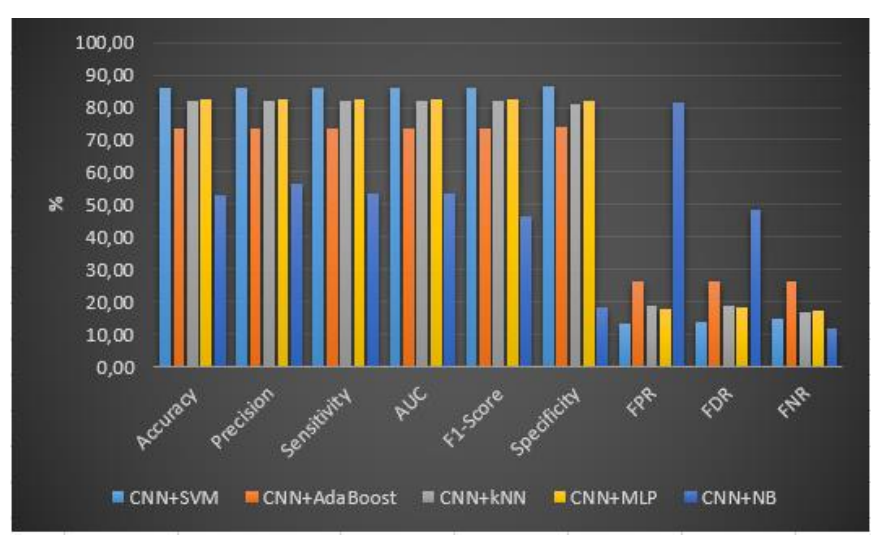

Figure 6 The Comparison of Accuracy, Precision, Sensitivity, AUC, F1-score, Specificity, FPR, FDR, and FNR for Each Model

A Confusion matrix is a metrics used to obtain the accuracy, precision, sensitivity, specificity, F1-score, FPR, FDR, and FNR of the model described in Equations (11-18). Table 3 shows true positives (TP), true negatives (TN), false positives (FP), and false negatives (FN) based on two classes (covid, normal).
Table 3 Confusion Matrix of the Observed Deep Learning Algorithms on Dataset

\begin{tabular}{|c|c|c|c|}
\hline \multirow{2}{*}{ Method } & \multirow{2}{*}{ Actual Classes } & \multicolumn{2}{|l|}{ Predicted } \\
\hline & & Covid & normal \\
\hline & Covid & $410(\mathrm{TP})$ & $71(\mathrm{FN})$ \\
\hline $\mathrm{CNN}+\mathrm{SVM}$ & Normal & $66(\mathrm{FP})$ & $422(\mathrm{TN})$ \\
\hline & Covid & 354 (TP) & $127(\mathrm{FN})$ \\
\hline CNN+AdaBoost & Normal & $128(\mathrm{FP})$ & $360(\mathrm{TN})$ \\
\hline & Covid & 401 (TP) & $80(\mathrm{FN})$ \\
\hline $\mathrm{CNN}+\mathrm{kNN}$ & Normal & $92(\mathrm{FP})$ & $396(\mathrm{TN})$ \\
\hline & Covid & $398(\mathrm{TP})$ & $83(\mathrm{FN})$ \\
\hline CNN+MLP & Normal & $88(\mathrm{FP})$ & $400(\mathrm{TN})$ \\
\hline $\mathrm{CNN}+\mathrm{NB}$ & $\begin{array}{l}\text { Covid } \\
\text { Normal }\end{array}$ & $\begin{array}{l}425 \text { (TP) } \\
399 \text { (FP) }\end{array}$ & $\begin{array}{l}56(\mathrm{FN}) \\
89(\mathrm{TN})\end{array}$ \\
\hline
\end{tabular}

To the best of our knowledge, the performance comparison of hybrid models (deep learning + machine learning) is limited on the combined image datasets. Table 4 shows the related studies which are using the same datasets. Wang et al. (2020) studied the Parallel Adapter algorithm to predict disease on the SARS-CoV-2 CT-scan dataset and obtained an accuracy of 82.13\%. Wang et al. (2020) also studied with SepNorm+Contrastive to predict disease on the COVID-CT dataset and obtained an accuracy of $78.69 \%$. Polsinelli et al. (2020) studied with CNN to predict disease on the COVID-CT dataset and obtained an accuracy of $83 \%$. Saeedi et al. (2020) studied with ResNet50V2 to predict disease on the COVID-CT dataset and obtained an accuracy of $84.03 \%$. Silva et al. (2020) studied EfficientNet Based Model to predict disease on combined image dataset and obtained an accuracy of 59.12\%. In this context, the $\mathrm{CNN}+\mathrm{SVM}$ hybrid model outperformed the best study ever reported in the literature in terms of performance metrics on the combined dataset.

Our proposed CNN method has fewer hidden layers in the proposed DL-based hybrid approach. We think that if $\mathrm{CNN}$ is trained with more neurons or more hidden layers or on a large amount of data, it can achieve higher accuracy results than $85.86 \%$. 
Table 4 Comparison with the Studies in the Literature

\begin{tabular}{|c|c|c|c|}
\hline Approach & Dataset & Method & Accuracy(\%) \\
\hline Polsinelli et al. (2020) & COVID-CT & CNN & 83 \\
\hline Silva et al. (2020) & SARS-CoV-2 CT-scan + COVID-CT & EfficientNet Based Model & 59.12 \\
\hline Wang et al. (2020) & SARS-CoV-2 CT-scan & Parallel Adapter & $\sim 82.13$ \\
\hline Wang et al. (2020) & SARS-CoV-2 CT-scan & Joint (Redesignt & $\sim 78.42$ \\
\hline Wang et al. (2020) & SARS-CoV-2 CT-scan & Single (COVID-Net) & $\sim 77.12$ \\
\hline Wang et al. (2020) & COVID-CT & Single (Redesign) & $\sim 77.07$ \\
\hline Wang et al. (2020) & COVID-CT & Joint (Redesign) & $\sim 69.67$ \\
\hline Wang et al. (2020) & COVID-CT & MS-Net & $\sim 76.23$ \\
\hline Wang et al. (2020) & COVID-CT & InceptionV3 & $\sim 78.69$ \\
\hline Saeedi et al. (2020) & COVID-CT & ResNet50V2 & $\sim 84.03$ \\
\hline Saeedi et al. (2020) & COVID-CT & ResNet50V1 & $\sim 73.72$ \\
\hline Saeedi et al. (2020) & COVID-CT & CNN+SVM & $\mathbf{8 5 . 8 6}$ \\
\hline Proposed & SARS-CoV-2 CT-scan + COVID-CT & & prospective \\
\hline
\end{tabular}

\section{Conclusions}

In this work, we proposed a framework for COVID-19 prediction. For the diagnosis, we applied DL-based hybrid architectures on CT combined datasets. CNN features were given to the machine learning classifiers (SVM, kNN, AdaBoost, MLP, NB) to measure the classification performance in a deep hybrid approach. CNN model consists of 4 convolutional layers, 4 max-pooling layers, and 4 dropout layers. These models automatically extract features from the input data and later generate COVID-19 predictions with machine learning techniques.

Model performance value is evaluated in terms of sensitivity, precision, accuracy, F1-score, AUC-score, specificity, FPR, FDR, and FNR. Experimental results are acquired with $\mathrm{CNN}+\mathrm{SVM}, \mathrm{CNN}+$ AdaBoost, $\mathrm{CNN}+\mathrm{kNN}$, $\mathrm{CNN}+\mathrm{MLP}$, and $\mathrm{CNN}+\mathrm{NB}$. With the developed CNN+SVM model, the highest accuracy rate of $85.86 \%$ is achieved.

The results can be improved by a larger CT dataset or by employing different $\mathrm{CNN}$ architectures. In the future, we will use different types of classifiers and different $\mathrm{CNN}$ architectures to improve the classification performance.

\section{References}

[1] KARAKUŞ, A. T.. "The Data Science Met with the COVID-19: Revealing the Most Critical Measures Taken for the COVID-19 Pandemic". Sakarya University Journal of Computer and Information Sciences, 3(3), 201-209, 2020.

[2] Wu X., et al.. "Deep learning-based multi-view fusion model for screening 2019 novel coronavirus pneumonia: a multicentre study". European Journal of Radiology, 109041, 2020.

[3] Jin C., et al.. "Development and Evaluation of an AI System for COVID-19 Diagnosis". medRxiv, 2020.

[4] Javaheri T., et al. "Covidctnet: An open-source deep learning approach to identify covid-19 using ct image". arXiv preprint arXiv:2005.03059, 2020.

[5] Jin S., et al.. "AI-assisted CT imaging analysis for COVID19 screening: Building and deploying a medical AI system in four weeks". medRxiv, 2020.

[6] Chen J., et al.. "Deep learning-based model for detecting 2019 novel coronavirus pneumonia on high-resolution computed tomography: a prospective study". MedRxiv, 2020.

[7] Ardakani A. A., Kanafi A. R., Acharya U. R., Khadem N., \& Mohammadi A. "Application of deep learning technique to manage COVID-19 in routine clinical practice using CT images: Results of 10 convolutional neural networks". Computers in Biology and Medicine, 103795, 2020.

[8] He X., Yang X., Zhang S., Zhao J., Zhang Y., Xing E., \& Xie P. "Sample-Efficient Deep Learning for COVID-19 Diagnosis Based on CT Scans". medRxiv, 2020.

[9] Wang S., et al. "A deep learning algorithm using CT images to screen for Corona Virus Disease (COVID-19)". MedRxiv, 2020.

[10]Song Y., et al. "Deep learning enables accurate diagnosis of novel coronavirus (COVID-19) with CT images". medRxiv, 2020.

[11]Zheng C., et al. "Deep learning-based detection for COVID19 from chest CT using weak label". medRxiv, 2020.

[12]Singh D., Kumar V., \& Kaur M. "Classification of COVID19 patients from chest CT images using multi-objective differential evolution-based convolutional neural networks". European Journal of Clinical Microbiology \& Infectious Diseases, 1-11, 2020.

[13]Farid A. A., Selim G. I., Awad H., \& Khater A. “A Novel Approach of CT Images Feature Analysis and Prediction to Screen for Corona Virus Disease (COVID-19)." Int. J. Sci. Eng. Res, 11(3), 1-9, 2020.

[14]Wang S., et al.. "A deep learning algorithm using CT images to screen for Corona Virus Disease (COVID-19)". medRxiv, 2020.

[15]Song Y., et al.. "Deep learning enables accurate diagnosis of novel coronavirus (COVID-19) with CT images". medRxiv, 2020.

[16]Yang X., et al. "COVID-CT-dataset: a CT scan dataset about COVID-19”. ArXiv e-prints, arXiv-2003, 2020.

[17]COVID-CT, https://github.com/UCSD-AI4H/COVID-CT (08.09.2020)

[18]Soares E., Angelov P., Biaso S., Froes M. H., \& Abe D. K. "SARS-CoV-2 CT-scan dataset: A large dataset of real patients CT scans for SARS-CoV-2 identification". medRxiv,2020.

[19]SARS-COV-2 Ct-Scan Dataset A large dataset of CT scans for SARS-CoV-2 (COVID-19) identification, 
https://www.kaggle.com/plameneduardo/sarscov2-ctscandataset (08.09.2020).

[20]Ciresan D. C., Meier U., Masci J., Gambardella L. M., \& Schmidhuber J. "Flexible, high performance convolutional neural networks for image classification". In Twenty-second international joint conference on artificial intelligence, 2011.

[21]Yildirim M., \& Cinar A. "A Deep Learning Based Hybrid Approach for COVID-19 Disease Detections". Traitement du Signal, 37(3), 461-468, 2020.

[22]Cortes C. "WSupport-vector network". Machine learning, 20, 1-25, 1995.

[23]Freund Y., \& Schapire R. E. "A decision-theoretic generalization of on-line learning and an application to boosting". Journal of computer and system sciences, 55(1), 119-139, 1997.

[24] Cover T., \& Hart P. "Nearest neighbor pattern classification". IEEE transactions on information theory, 13(1), 21-27, 1967.

[25]Witten I. H., \& Frank E. "Data mining: practical machine learning tools and techniques with Java implementations". Acm Sigmod Record, 31(1), 76-77, 2002.

[26]Russell S., \& Norvig P. "Artificial intelligence: a modern approach". 2002.

[27]Polsinelli M., Cinque L., \& Placidi G. "A Light CNN for detecting COVID-19 from CT scans $\mathrm{f}$ the chest. arXiv preprint arXiv:2004.12837”. 2020.

[28]Silva P., Luz E., Silva G., Moreira G., Silva R., Lucio D., \& Menotti D. "COVID-19 detection in CT images with deep learning: A voting-based scheme and cross-datasets analysis". Informatics in Medicine Unlocked, 20, 100427, 2020.

[29]Wang Z., Liu Q., \& Dou Q. "Contrastive Cross-Site Learning With Redesigned Net for COVID-19 CT Classification". IEEE Journal of Biomedical and Health Informatics, 24(10), 2806-2813, 2020.

[30] Saeedi A., Saeedi M., \& Maghsoudi A. "A novel and reliable deep learning web-based tool to detect COVID-19 infection form chest CT-scan". arXiv preprint arXiv:2006.14419, 2020. 